Alignment error evaluation of the dial pattern

This content has been downloaded from IOPscience. Please scroll down to see the full text. 1998 J. Micromech. Microeng. 8239

(http://iopscience.iop.org/0960-1317/8/3/010)

View the table of contents for this issue, or go to the journal homepage for more

Download details:

IP Address: 140.113.38.11

This content was downloaded on 28/04/2014 at 11:24

Please note that terms and conditions apply. 


\title{
Alignment error evaluation of the dial pattern
}

\section{Ying-Hwi Chang and Yu-Chung Huang}

Institute of Electronics, National Chiao Tung University, 1001 Ta Hsueh Road, Hsinchu Taiwan, Republic of China $\dagger$

Received 15 January 1998, accepted for publication 19 March 1998

\begin{abstract}
To achieve smooth etched sidewall surface at minimal time expense and reduce undercut etching are basic disciplines of the task of accurate alignment. When aligning etch-masks to $\langle 110\rangle$ crystal orientation on a (100) Si wafer, a consideration is proposed by Schröder who uses a dial pattern for pre-etching to determine $\langle 110\rangle$ crystal orientation. But that performance depends strongly on the resolution of the mask pattern generator. In this paper, we analyse Schröder's method by mathematical modeling and computer simulation. Finally, we propose an improvement for the Schröder limitation according to our analytic results.
\end{abstract}

\section{Introduction}

Having accurately aligned etch-masks to $\langle 110\rangle$ crystal orientation on a (100) Si wafer can save time to obtain a smooth etched sidewall surface at minimal time expense and reduce undercut etching. Several methods for alignment of mask patterns to crystal orientation have been proposed already [1-5]. Schröder [1] proposed an improved method from Ensell's research [2,3] to accomplish this task. Instead of the sophisticated approach [4], his method can be used together with an optical microscope to find the $\langle 110\rangle$ crystal orientation without any other special equipment. Schröder's works use the dial pattern with an origin at the center of the wafer and a radius slightly smaller than the wafer. The small circles of the dial pattern are separated equally with an angular pitch. The $X$-axis of the mask is aligned to be parallel with the edge of the primary flat. The dial pattern spans $\pm 2^{\circ}$, that is enough to account for the aligning error and the variation of the $\langle 110\rangle$ orientation. The patterned wafer is then etched by an anisotropic etching solution such as $\mathrm{KOH}$ and produces square pits for each small circle on the mask as shown in figure 1 [2,3]. A target etched square can be found according to some criterion. The label besides the target etched square indicates the error angle between the mask coordinate system and the $\langle 110\rangle$ orientation.

In our studies, we found that the performance of Schröder's method depends strongly on the accuracy of the coordinates of the small circles' origins of the dial pattern. Moreover, that is limited by the resolution of the mask pattern generator. The typical resolution of the mask pattern generator for MEMS applications is about $0.1 \mu \mathrm{m}$ [6]. But this is large enough to significantly degrade the accuracy of Schröder's method.

\footnotetext{
$\dagger$ Tel: +886-3-5712121 ext. 54208. Fax: +886-3-5724361 e-mail: u7911505@cc.nctu.edu.tw.
}

In this paper, we first analyse Schröder's method by mathematical modeling and computer simulation. Finally, we propose an improvement for the Schröder limitation.

Referring to figure 1, the notations defined below are used in this work.

$\theta$ : the error angle between the mask coordinate system and $\langle 110\rangle$ orientation.

$\mathrm{R}_{\phi}$ : the etched square at angle $\phi$.

$\phi_{T}$ : the $\phi$ that is the closest to $\theta$ found from the etched squares.

$\mathrm{C}_{\phi}$ : the central point of $\mathrm{R}_{\phi}$.

$r_{O}$ : the radius of the dial pattern.

$r_{C}$ : the radius of the small circles for the dial pattern.

$D_{P L}(\mathrm{P}, \mathrm{L})$ : distance from point $\mathrm{P}$ to the straight line $\mathrm{L}$.

$\langle\cdot\rangle$ : round off a value.

$H_{\phi} / T_{\phi}$ : spacings between etched square pits.

\section{Modeling}

Referring to figure 1 , the etched square $\mathrm{R}_{\phi_{T}}$ is the target square that should be found by the microscopic inspection and comparison of the etched squares. Then $\phi_{T}$ can be identified from the label beside $\mathrm{R}_{\phi_{T}}$. For an etched square $\mathrm{R}_{\phi}, H_{\phi}$ and $T_{\phi}$ can be found from the microscopic inspection. The mathematical equations for $H_{\phi}$ and $T_{\phi}$ were derived according to figure 1 as shown in (1)-(2).

$$
\begin{aligned}
H_{\phi}= & \left(D_{P L}\left(\mathrm{C}_{\phi-\delta \phi}, \mathrm{L}_{\langle\overline{1} 10\rangle}\right)-r_{C}\right)-\left(D_{P L}\left(\mathrm{C}_{\phi}, \mathrm{L}_{\langle\overline{1} 10\rangle}\right)-r_{C}\right) \\
& =r_{O} \cos (\phi-\delta \phi-\theta)-r_{O} \cos (\phi-\theta) \\
& =2 r_{O} \sin \left[(\phi-\theta)+\frac{\delta \phi}{2}\right] \sin \left(\frac{\delta \phi}{2}\right) \\
T_{\phi}= & \left(D_{P L}\left(\mathrm{C}_{\phi+\delta \phi}, \mathrm{L}_{\langle 110\rangle}\right)-\left(D_{P L}\left(\mathrm{C}_{\phi}, \mathrm{L}_{\langle 110\rangle}\right)-2 r_{C}\right)\right. \\
& =r_{O} \sin (\phi-\delta \phi-\theta)-r_{O} \sin (\phi-\theta)-2 r_{C} \\
& =2 r_{O} \cos \left[(\phi-\theta)+\frac{\delta \theta}{2}\right] \sin \left(\frac{\delta \phi}{2}\right)-2 r_{C}
\end{aligned}
$$




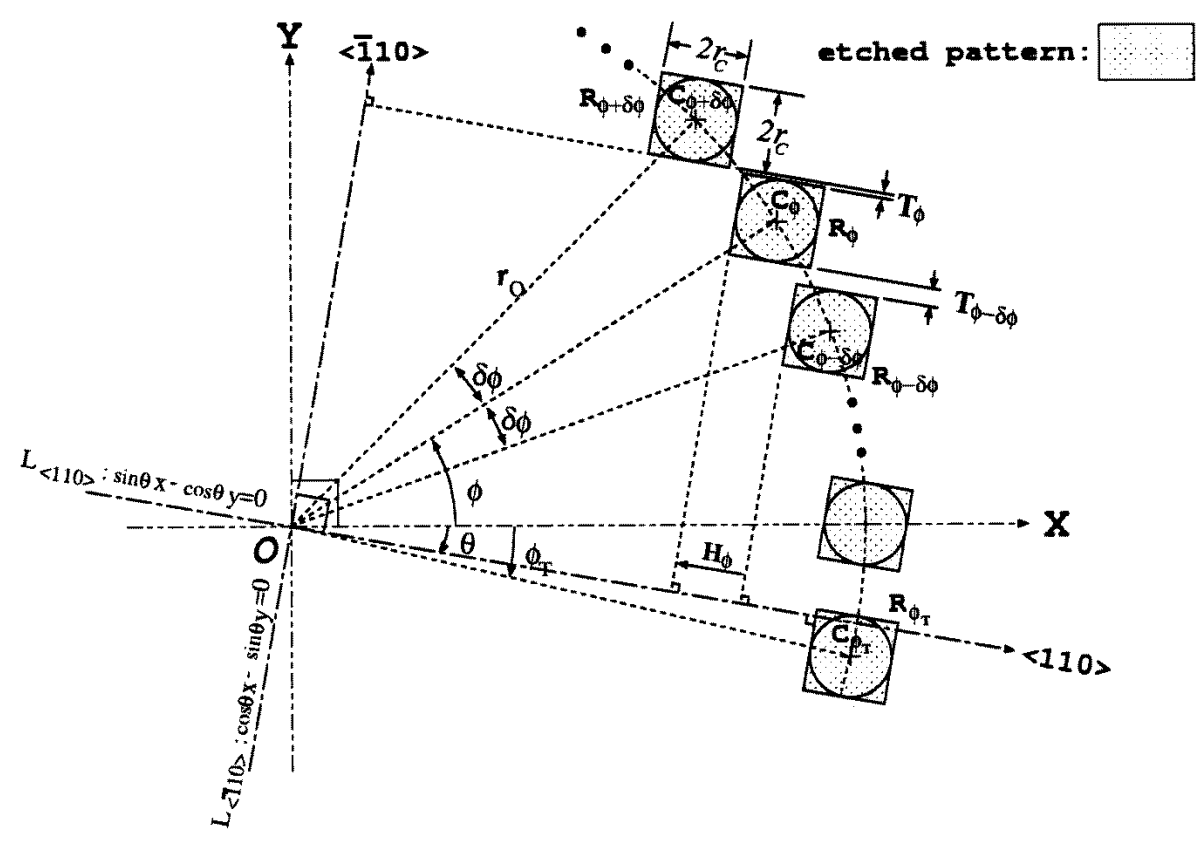

Figure 1. Illustration of the anisotropic etched patterns on a (100) Si wafer patterned by the pre-etching mask with the dial pattern.

Three kinds of criterion by comparing $H_{\phi}, T_{\phi}$ and $T_{\phi-\delta \phi}$ can be applied to determine $\mathrm{R}_{\phi_{T}}$. Criterion 1 is that the inner edge of $R_{\phi_{T}}$ is the farthest away from the origin of the dial pattern $\mathrm{O}$. This criterion can be applied by finding the last $\mathrm{R}_{\phi}$ of which $H_{\phi}$ is positive from $\phi=2^{\circ}$ counting back. Criterion 2 is that $T_{\phi_{T}}-T_{\phi_{T}-\delta \phi}$ is minimum for all etched squares. Criterion 3 is that $T_{\phi_{T}}+T_{\phi_{T}-\delta \phi}$ is maximum for all etched squares. The two equations for $T_{\phi}-T_{\phi-\delta \phi}$ and $T_{\phi}+T_{\phi-\delta \phi}$ used for criterion 2 and criterion 3 were found from (2) as shown in (3) and (4).

$$
\begin{aligned}
& T_{\phi}-T_{\phi-\delta \phi}=4 r_{O} \sin (\phi-\theta) \sin ^{2}\left(\frac{\delta \phi}{2}\right) \\
& T_{\phi}+T_{\phi-\delta \phi}=4 r_{O} \cos (\phi-\theta) \cos \left(\frac{\delta \phi}{2}\right) \sin \left(\frac{\delta \phi}{2}\right)
\end{aligned}
$$

All circles will be assumed to be identical but individually shifed from the perfect circle to the closest grid position according to the resolution of the mask pattern generator. The finite resolution effect of the mask pattern generator was modeled by replacing $\mathrm{C}_{\phi}$ with $\mathrm{C}_{\phi}^{\prime}$ for re-derivation of (1) and (2). $H_{\phi}$ and $T_{\phi}$ were derived again as shown in (5) and (6).

$$
\begin{aligned}
H_{\phi}= & \left(D_{P L}\left(\mathrm{C}_{\phi-\delta \phi}^{\prime}, \mathrm{L}_{\langle\overline{1} 10\rangle}\right)-r_{C}\right)-\left(D_{P L}\left(\mathrm{C}_{\phi}^{\prime}, \mathrm{L}_{\langle\overline{1} 10\rangle}\right)-r_{C}\right) \\
& =\cos \theta\left[\left\langle r_{O} \cos (\phi-\delta \phi)\right\rangle-\left\langle r_{O} \cos \phi\right\rangle\right] \\
& +\sin \theta\left[\left\langle r_{O} \sin (\phi-\delta \phi)\right\rangle-\left\langle r_{O} \sin \phi\right\rangle\right] \\
T_{\phi}= & \left(D_{P L}\left(\mathrm{C}_{\phi+\delta \phi}^{\prime}, \mathrm{L}_{\langle 110\rangle}\right)-\left(D_{P L}\left(\mathrm{C}_{\phi}^{\prime}, \mathrm{L}_{\langle 110\rangle}\right)-2 r_{C}\right)\right. \\
& =\cos \theta\left[\left\langle r_{O} \sin (\phi+\delta \phi)\right\rangle-\left\langle r_{O} \sin \phi\right\rangle\right] \\
& -\sin \theta\left[\left\langle r_{O} \cos (\phi+\delta \phi)\right\rangle-\left\langle r_{O} \cos \phi\right\rangle\right]-2 r_{C}
\end{aligned}
$$

\section{Simulation results}

According to the model derived from the previous section and considering $100 \mathrm{~mm}$ wafers for processing, some simulation works were completed in order to explain the effects of the finite resolution of the mask pattern generator on Schröder's method. Other interferences such as measurement error while collecting data, uncertainty in the mask pattern generator etc are not included in the simulation.

For the ideal case, i.e. no round-off effect on the coordinate of $\mathrm{C}_{\phi}$, the simulation of the relations between $\phi_{T}$ and $\theta$ was done for the three methods described in section 2 with $r_{O}=50000 \mu \mathrm{m}$ and $\delta \phi=0.05^{\circ}$. The readout errors are identical for these three criteria as shown in figure 2(a). The accuracy is $\pm 0.025^{\circ}$, i.e. $0.5 \delta \phi$. Whereby rounding off the coordinates of $\mathrm{C}_{\phi}$ at $0.1 \mu \mathrm{m}$, the readout errors are shown in figure 2(b) for criterion 1, figure 2(c) for criterion 2 and figure 2(d) for criterion 3. The readout errors as shown in figure 2 for criterion 1, 2 and 3 in the round-off case are 3.4, 160 and 80 times larger than the ideal case respectively.

The simulation result of the relations between the accuracy and round-off precision for the three criteria with $r_{O}=50000 \mu \mathrm{m}$ and $\delta \phi=0.05^{\circ}$ is plotted in figure 3. As indicated in figure 3 , criterion 1 is the most insensitive to the round-off precision among the three criteria.

A basic approach to reduce the round-off effect on the coordinate of $\mathrm{C}_{\phi}$ is to enlarge its dimension. This can be improved by increasing $r_{O}$. The simulation of the relations between the accuracy and $r_{O}$ was made for the three criteria with round-off precision $0.1 \mu \mathrm{m}$ and $\delta \phi=0.05^{\circ}$ and the result is shown in figure 4. As indicated in figure 4, increasing $r_{O}$ has significant improvements on the accuracy 

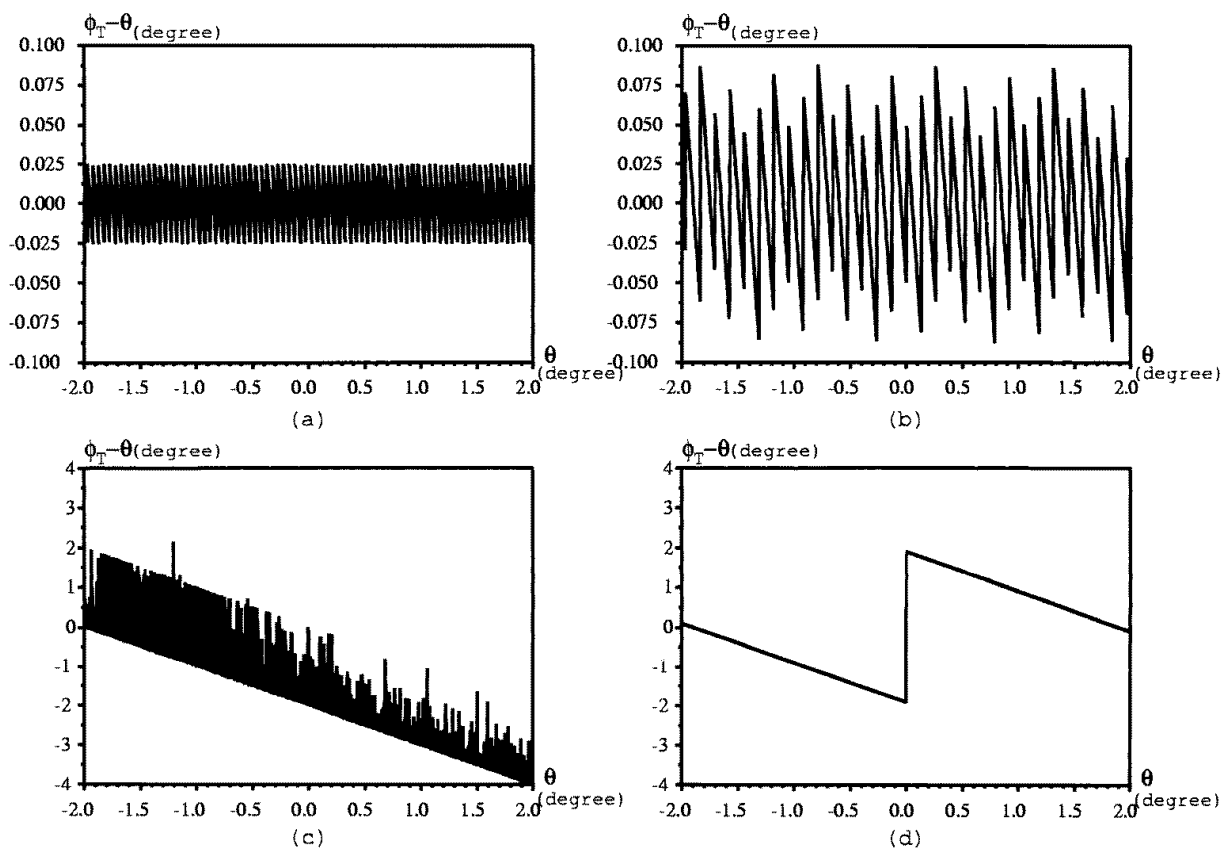

Figure 2. Simulation results: readout error $\left(\phi_{T}-\theta\right)-\theta$ plots with $r_{O}=50000 \mu \mathrm{m}$ and $\delta \phi=0.05^{\circ}$ for (a) no round-off and all three criteria, (b) round-off precision $0.1 \mu \mathrm{m}$ and criterion 1, (c) round-off precision $0.1 \mu \mathrm{m}$ and criterion 2 and (d) round-off precision $0.1 \mu \mathrm{m}$ and criterion 3.

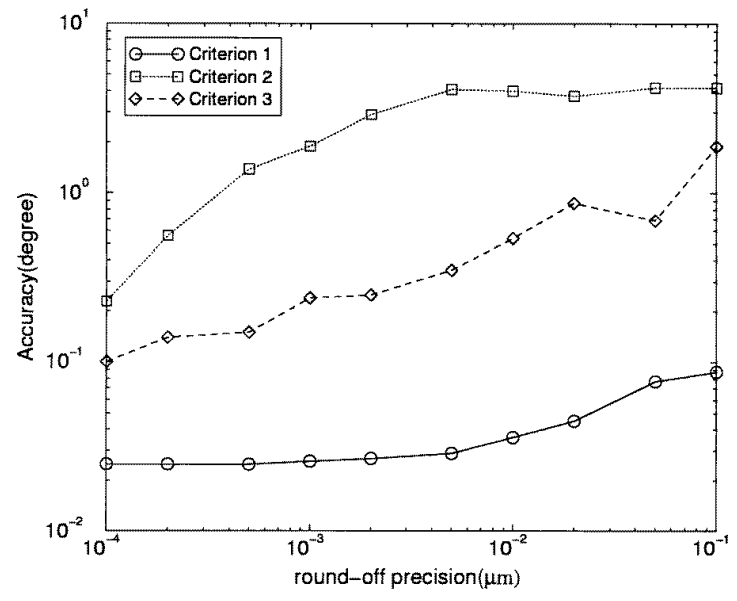

Figure 3. Simulation result: accuracy-round-off precision plot with $r_{O}=50000 \mu \mathrm{m}$ and $\delta \phi=0.05^{\circ}$.

for all of the three methods. Besides, similar simulation results were found for ceiling or flooring instead of roundoff for all the simulation cases described above.

\section{Conclusion}

The finite resolution of the mask pattern generator has remarkable influence on the accuracy of Schröder's method. As shown in figure 3, improving the situation by using a more precise mask pattern generator is insignificant even with resolution as small as $0.025 \mu \mathrm{m}$ [7]. The accuracy of Schröder's method, as depicted in figure 4, can be improved by applying the criterion 1 described in section 2 to find

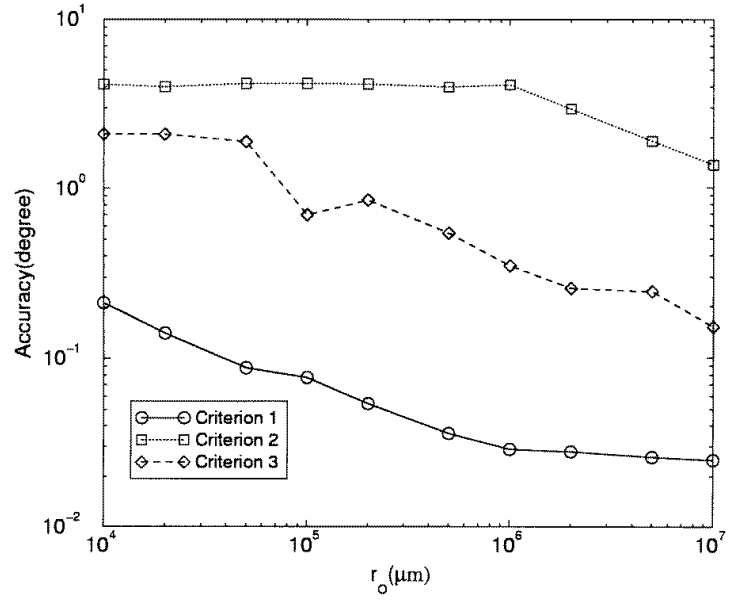

Figure 4. Simulation result: accuracy- $r_{O}$ plot with round-off precision $0.1 \mu \mathrm{m}$ and $\delta \phi=0.05^{\circ}$.

$\mathrm{R}_{\phi_{T}}$, and increasing $r_{O}$. The origin of the dial pattern can be chosen such that the dial pattern can fit into the required area on the wafer besides choosing the wafer's center as the origin of the dial pattern in Schröder's works. In this way, the dial pattern can not be used as alignment keys for the subsequent mask. Therefore, another pattern should be added as alignment keys such as Schröder's pattern or Ensell's pattern [1-3]. Moreover, the choice of $r_{O}$ should be compromised with pattern area and etching time since both of them increase as $r_{O}$ increases. 


\section{References}

[1] Schröder H, Dorsch O and Obermeier E 1996 An improved method to align etchmasks to the $\langle 110\rangle$ crystal orientation Micro System Technologies '96 ed H Reichl and A Heuberger (Berlin: Vde) pp 651-5

[2] Ensell G 1995 Alignment of mask patterns to crystal orientation Transducers '95, 8th Int. Conf. on Solid-State Sensors and Actuators, and Eurosensors IX, Tech. Digest Papers (Stockholm) pp 186-9

[3] Ensell G 1996 Alignment of mask patterns to crystal orientation Sensors Actuators A 53 345-8

[4] Steckenborn A et al 1991 High precision wafer orientation for micromachining Micro System Technologies '91 ed R Kahn and H Reichl (Berlin: Vde) pp 467-71

[5] Vangbo M and Bäcklund Y 1996 Precise mask alignment to the crystallographic direction of silicon wafers using wet anisotropic etching J. Micromech. Microeng. 6 279-84

[6] Heidelberg Instruments Mikrotechnik Specifications for mask pattern generator 1997 DWL 66

[7] Gerber Specifications for mask pattern generator MaskWrite 800 and 1550 